\title{
On the Effect of Human Body Shape on Fashion Pattern Making Hong Cai
}

\author{
Clothing Department, Wuxi Institute of Arts and Technology, Wuxi, 214206, China
}

Keywords: fashion pattern making; human body shape; structure; effect; analysis

\begin{abstract}
This paper takes the fashion pattern making as object of study, focuses on consideration of the human body structure, starts from the effect of human neck, shoulder, chest, back and waist on the fashion pattern making, proceeds with detailed analysis and elaboration on the issue centering on the effect of human body shape on fashion pattern making, and hereby demonstrates the important position and critical importance of the cognition and high attention to human body shape in the processes of improving the quality of fashion pattern making and optimizing the comfortableness and artistry of clothing and dressing. What is human body shape? In brief, human body shape is a general term describing the human shape and the muscular and bone tissues that can affect the shape. At the current stage, most people dissatisfy with their body shapes. From the perspective of clothing structure design, one of the most critical issues at the current stage for the staff related to fashion design and fashion pattern making is to produce finished clothing products that fully follow the general proportion and regularity of human body structure, cover the shortages and fully reveal the advantages of human body shape, and have different design styles and unique arts and colors. From this point of view, the staff involved in fashion design and pattern making shall not only fully understand the characteristics and structure of human body, but also achieve the deepening and sublimation of clothing's own aesthetic value on the basis of considering the dynamic and static characteristics of human body and meeting clothing's basic function of covering the body and with the help of embodying the external beauty. This paper tries to make a detailed analysis and description on such issue.
\end{abstract}

\section{Analysis on the effect of human neck on fashion pattern making}

From the perspective of human body structure, neck is the intermediate connecting structure of head structure and body structure. From the horizontal point of view, the neck structure is similar to a circular structure; from the longitudinal point of view, the neck structure presents a cone-shaped structure with thick bottom and thin top; from the integral point of view, the neck structure presents the trend of certain front inclination, which determines the design style of clothing collar. Meanwhile, the basic shape of neck structure has certain relation to human gender. Generally, the cross-section shape of men's neck structure looks like a peach, while that of women's neck structure looks like a persimmon; such difference can be the most basic reference for the design of collar radian during fashion pattern making. In the meantime, the particularity of the human body structure determines that the position of the collar band edge should be at the front upper boundary of the neck, and that the lower part should be above the clavicle, embodying a cylindrical structure with thick bottom and thin top in an integral way. The three-phase position connection of front neck point, rear neck point and inclined neck point during fashion pattern making can form a basic neckline shape where the rear side is wide, high and flat and the front side is narrow, low and curved. From the perspective of the analysis on relevant practice and research results, the determination of collarband position during fashion pattern making relates directly to the question whether the overall fashion design can improve the sense of beauty under the premise of guaranteeing the comfortableness of dressing. From the perspective of current stage of fashion design and pattern making, the collar shape design is presenting a trend of diversified development; however, the key point of collar design is to design it on the basis of characteristics of human neck. At the same time, considering the achievement of spinning movement at the neck position, the top-to-bottom and front-to-rear cutting way should be considered for neckline, so as to ensure 
comfortableness of dressing.

\section{Analysis on the effect of human shoulder on fashion pattern making}

From the perspective of human body structure, the human shoulder position basically presents a slight tilt state. From the look-down angle of view, such slight tilt state shows a slight forward-curved shape. Like the neck position in the human body structure, the tilting degree of human shoulder also has certain difference according to the human gender. Generally, the tilting degree of a normal man's shoulder is 18o, while that of a woman's shoulder is 20o. During the research and analysis on characteristics of human shoulder structure, another key parameter is also involved, namely, off-the-shoulder difference. However, it is discovered from practice and research results that the parameter of off-the-shoulder difference reflected by human body structure itself has, to some extent, certain relation to the sizes of human shoulder blade and chest; therefore, it is impossible to clearly limit the off-the-shoulder difference to a specific numerical range at current stage. Meanwhile, if the shoulder position is designed with shoulder pads during fashion pattern making, attention should also paid to the effective thickness increased due to adding such shoulder pads when considering the shoulder tilting degree of upper clothing during pattern making and forming of such upper clothing. From this point of view, the off-the-shoulder degrees involved in the process of fashion pattern making will also have certain difference, according to the different shoulder tilting degrees reflected by the human body structure; in this process, the difference existing between shoulder width and front chest breadth and between shoulder width and back across is called back across-shoulder width difference. To some extent, the back across-shoulder width difference is generally the most fundamental reason for the situation that the rear shoulder tilting degree is obviously less than the front shoulder tilting degree during pattern making of upper clothing. Besides, in this process, the particularity of human body structure makes the front chest breadth and back across reflect certain proportion of broadening when the chest circumference is increasing. On the basis of this factor, the pattern of externally-curved front shoulder line and internally-curved rear shoulder line should be adopted during fashion pattern making.

\section{Analysis on the effect of human chest on fashion pattern making}

From the perspective of human body structure, the chest structure consists of partial spine structure, sternum and twelve pairs of ribs; the cross-section shape of such structure is of a cylindrical shape. In terms of the overall body structure, the chest structure is the plumpest area, is the most fundamental reference for measuring the basic parameters of the chest during fashion design and pattern making, and occupies an extremely key and central position in the whole process of clothing structure design and fashion pattern making. Meanwhile, the gender difference generally determines the chest shape difference. In general, the most muscular area of a man's chest is the pectoralis major area, which presents a certain raised state relative to the overall body structure. In the meantime, a woman's overall chest structure shows a conical raised state relative to the rear tilting of the body. Especially for adult women, the development of the chest structure is the most obvious in the overall body structure. From the perspective of fashion pattern making, the most severe challenge faced by fashion pattern making is, generally, how to fully embody female's line of beauty in terms of female's three-dimensional curve with significant changes and on the basis of handling the shape design of breast and other positions. At the same time, with the continuous increase of female's age, the breasts will have certain degree of looseness and prolapse. From the perspective of fashion pattern making, the fashion design and pattern making, especially the design and pattern making of women's clothing, should make clear the downward directivity of women's body structure from the second rib to the sixth or seventh rib and the positions of nipples. Especially for the cutting of women's clothing, one of the most basic issues is to consider the exact positions and protuberant degree of breasts. With the support of the current stage of technical conditions for fashion pattern making, the pattern making demands of female's chest structure are often achieved with pleats and darts, while that of male's chest structure is generally achieved with 
splitting form. The application of such fashion pattern making forms can not only meet the basic physiological needs of men and women, but also accord with the development requirements of the new era of clothing design artistry. Combining the effective utilization of darts, the three-dimensional state and concave-convex changes of chest position design of clothing can be also embodied in a sufficient and real way.

\section{Analysis on the effect of human back on fashion pattern making}

The different characteristics of back shape in the human body structure will directly result in the difference of front and rear pieces during pattern making of men's and women's clothing. Firstly, in terms of women, the women's unique body physical characteristics make the rear piece in the back structure be about $0.5 \mathrm{~cm}$ shorter than the front piece, which determines that the pleating treatment of $3 \mathrm{~cm}$ is required for the axilla position of women's clothing during fashion pattern making; besides, in terms of men, the back thickness of a normal man is obviously greater than that of a woman, and the problem of forward body tilting to some extent makes the rear piece of back structure be about $2.5 \mathrm{~cm}$ longer than the front piece. The back position is designed with slotting for some men's clothing during pattern making, of which the key purpose is to ensure that the rear piece of finished clothing is more fitting in the dressing state and can fully and effectively embody the structural features and beauty of human body. Meanwhile, the fashion pattern making carried out on the basis of considering the factor of human back structure should also take into full consideration the satisfiability of human body motion. In the process of fashion design and cutting, full attention should be paid to the combination of human body features and corresponding laws of motion, and all kinds of motion forms of human body should be met on the basis of not affecting the overall dressing effect of the clothing. In this process, full attention should be also paid to the effective setting of looseness for back side position of fashion pattern making; in the actual application, the end point is to satisfy the front lifting of upper arm motion, and effective limitation should be carried out for backswing.

\section{Analysis on the effect of human waist on fashion pattern making}

From the perspective of the overall body structure, the waist position is the thinnest position in the whole body structure. Meanwhile, it is a structure that is the most prominent in terms of activity range and flexibility. From the perspective of fashion pattern making, the human waist structure is also one of the key areas that shape and deepen the beauty. From the standpoint of research on human body structure, the waist structure is not strictly at the level position in the thinnest-position state, but at a "high at rear side and low at front side" fluctuating state. In the process of fashion pattern making, it is required to accomplish the design and attended operation of clothing products in accordance with the specific activity range of human body and the seasonal characteristics. As far as the specific waist structure is concerned, its major direction of motion is the forward motion; on the basis of this analysis, the waisted shape is often required at the waist position during fashion pattern making. On this basis, the margin of $2 \mathrm{~cm}$ is to be lengthened at the rear waist position of the cut piece, to ensure that the fashion pattern making at the waist position can match with the basic shape of human body. A more important point is that the error of measuring and cutting sizes at the waist position should be properly controlled and excessively waisted treatment should be avoid at the waist area, so as to avoid adverse effect on normal motion of the waist; generally, certain margin should be reserved to guarantee comfortable motion.

\section{Conclusion}

In our country, the clothing industry during the current stage of socialist market economy development is gradually moving towards the road of branding development; therefore, the significance of physical characteristics in the fashion design and pattern making is further highlighted. In a word, this paper has made a brief analysis and description about the effect of 
human body shape on fashion pattern making in the hope of drawing special attention of relevant personnel.

\section{References}

[1] Zhong Li and Hu Xuemei, The Development Prospect of Three-dimensional CAD in Fashion Pattern Making, [J]. Journal of Chengdu Textile College, 2009.26.(03).18-19.

[2] Su Junqiang, Tian Tian, Li Hong, etc., Professional Innovation Education Based on Knowledge Fermentation _ Taking Fashion Pattern Making and Technology Specialty as Examples, [J]. Journal of Taiyuan City Vocational College, 2010.(11).1-2.

[3] Huang Zhenzhen, Wang Xiaoyun, Zhang Hongzhi, etc., Forecast on Human Body Parameter Measurement with Sizes of Small Human Body Sample, [J]. Journal of Tianjin Polytechnic University, 2010.29.(02).34-38.

[4] Hu Changpeng, Zhang Jujian, Liu Ruipu, etc., Research on Automatic Drawing of Suit Pattern Based on Secondary Development with AutoCAD, [J]. Journal of Zhejiang Fashion Institute of Technology, 2010.09.(04).21-25.

[5] Mu Hong and Liu Guolian, Research on Structural Treatment Methods at Shoulder Line and Lapel Positions of Men's Suits Made of Gingham, [J]. Journal of Suzhou University (engineering version), 2005.25.(02).63-66.

[6] Yu Linyan and Zhang Zufang, Size Design of Read-to-wear Clothing for the Medium-elderly Women — Research on Improving the Body Shape Coverage with Clothing, [J]. Journal of Xi'an Polytechnic University, 2003.17.(02).118-122.

[7] Yu Xiaokun and Wang Jianping, Matching Relation between Human Body Shape and Clothing Size and Its Application in E-commerce, [J]. Journal of Donghua University (natural science version), 2003.29.(01).43-47.76.

[8] Xu Yichao, Chen Bin, Ding Yongsheng, etc., Clothing Fit Evaluation Oriented Virtual Human Modeling and Body Shape Forecast, [J]. Computer Engineering and Application, 2008.44.(01). 232-234. 